Cahiers de la recherche sur les droits fondamentaux

$12 \mid 2014$

Droit et psychiatrie

\title{
La loi du 5 juillet 2011 sur les soins psychiatriques : les positions de l'UNAFAM
}

Denis Malo

\section{(2) OpenEdition}

Édition électronique

URL : http://journals.openedition.org/crdf/1892

DOI : $10.4000 /$ crdf.1892

ISSN : 2264-1246

Éditeur

Presses universitaires de Caen

Édition imprimée

Date de publication : 1 décembre 2014

Pagination : 53-56

ISBN : 978-2-84133-507-7

ISSN : $1634-8842$

Référence électronique

Denis Malo, "La loi du 5 juillet 2011 sur les soins psychiatriques : les positions de l'UNAFAM », Cahiers de la recherche sur les droits fondamentaux [En ligne], 12 | 2014, mis en ligne le 01 octobre 2015,

consulté le 11 février 2020. URL : http://journals.openedition.org/crdf/1892 ; DOI : 10.4000/crdf.1892

Cahiers de la recherche sur les droits fondamentaux 


\title{
La loi du 5 juillet 2011 sur les soins psychiatriques: les positions de I'UNAFAM ${ }^{1}$
}

\author{
Denis MALO \\ Membre bénévole de la délégation de I'Union nationale des amis ou familles de malades psychiques (UNAFAM) Calvados
}

\author{
I. Avant la loi, quelques remarques chronologiques \\ II. La loi et sa perception par I'UNAFAM \\ III. L'UNAFAM et la loi du 27 septembre 2013 modifiant la loi du 5 juillet 2011
}

Au nom de l'UNAFAM, tous mes remerciements aux organisateurs de ce colloque qui permet aux proches des patients d'exprimer leur point de vue: les bénévoles de l'UNAFAM n'ont ni de légitimité juridique ni de légitimité psychiatrique: seulement celle de «veilleur au quotidien». Mon intervention s'appuie donc sur le ressenti de l'entourage et de son expérience quant à la promulgation et l'application de la loi de 2011 et de ses suites.

\section{Avant la loi, quelques remarques chronologiques}

La loi dont nous parlons était dans les cartons depuis 1995 (la loi de 1990 étant en principe révisable tous les cinq ans). Le rapport «Strohl $»^{2}$ préconisait en 1995 un système d'urgences à compétence psychiatrique et l'obligation de soins (que l'on peut d'ailleurs entendre de deux manières: obligation pour le patient mais également pour la société, via les soignants). En 2010, une association d'usagers a enclenché une démarche auprès du Conseil constitutionnel: le maintien de l'hospitalisation sans consentement du patient sans l'intervention d'un juge a alors été reconnu anticonstitutionnel ${ }^{3}$. La volonté d'établir une nouvelle loi a ensuite ressurgi lors de drames très médiatisés (l'UNAFAM n'en a pas fini de protester contre une médiatisation indigne qui désinforme régulièrement les citoyens sur la nature exacte des troubles psychiques...). Engagée dans les discussions qui avaient précédé, l'UNAFAM a alors souhaité, très isolée, apporter son point de vue dans l'élaboration du projet de loi en s'appuyant sur l'une de ses principales revendications: obtenir un dispositif de réponse aux urgences psychiatriques.

\section{La loi et sa perception par l'UNAFAM}

Sont énoncés ci-dessous les points de cette loi apparus à l'UNAFAM comme sources de progrès, même s'il faut observer aujourd'hui encore une grande vigilance à l'égard de son application.

1. Les propos de cette intervention reprennent très largement un article de la revue de l'UNAFAM: B. Escaig, J. Canneva, «Le Juge arbitre, l'Hospitalier et le Malade psychique», Un autre regard, $\mathrm{n}^{\circ}$ 3, 2011, p. 1-5.

2. Rapport qui réunissait magistrats, psychiatres et usagers, dont l'UNAFAM; A. Lazarus, H. Strohl, Une souffrance qu'on ne peut plus cacher, rapport du groupe de travail «Ville, santé mentale, précarité et exclusion sociale», Paris, La documentation française, 1995.

3. Voir CC, déc. no 2010-71 QPC du 26 novembre 2010, M"le Danielle S. 
Lors de la demande d'une hospitalisation par un tiers, la famille demande des soins et non une hospitalisation. La demande a donc le caractère d'un signalement, c'est-à-dire d'un besoin de soins et non d'un enfermement comme par le passé: ce sont aux médecins de décider s'il s'agira de soins libres ou sans consentement, ambulatoires ou en hospitalisation complète, la personne malade étant conduite à l'hôpital dans un "centre d'évaluation " pour soixante-douze heures au plus.

Le traitement de l'urgence psychiatrique: la loi impose aux agences régionales de santé (ARS) d'organiser un dispositif d'urgence capable d'assurer aux personnes malades des «soins appropriés» et le cas échéant de faire assurer leur transport vers l'hôpital.

Autre innovation appréciable pour l'UNAFAM: un directeur d'hôpital peut admettre un patient en soins psychiatriques sans la demande d'un tiers (quand la famille est absente ou en impossibilité) quand il existe un péril imminent pour la santé de la personne (péril constaté par médecin extérieur à l'hôpital). L'entourage en est informé dans les vingt-quatre heures.

Les soins sans contentement en ambulatoire peuvent également, selon l'UNAFAM, constituer une amélioration de la qualité des soins. Le patient n'est pas ou plus appelé à résider à l'hôpital mais doit respecter un programme de soins contracté avec le psychiatre. S'il y a rupture du contrat par le patient, le psychiatre le constate et propose immédiatement au directeur de l'hôpital une hospitalisation complète, le patient ayant été informé de cette éventualité dès l'établissement de son programme de soins. Le directeur de l'établissement accède à la demande du psychiatre en vertu d'une simple modification de la modalité des soins, sans besoin d'une demande familiale, de certificats médicaux ou d'évaluation.

Le programme de soins figure sur un document établi par le psychiatre après avis du patient: cette nouvelle pratique a en quelque sorte "légalisé» la sortie d'essai souvent prolongée durant de longues périodes, voire même des années dans le passé.

Désormais, toute personne en hospitalisation complète sans son consentement ne peut y rester durablement sans que le juge des libertés et de la détention (JLD) ait statué. Le juge dispose d'un avis conjoint rendu par deux psychiatres de l'hôpital ou par un collège de soignants (selon les diverses modalités de la durée de l'hospitalisation). La loi stipule dans son article 6 (nouvel art. L. 3211-12-2.-I du Code de la santé publique) que «le juge après débat contradictoire, statue publiquement». L'UNAFAM y voit la source de divers problèmes:

- la présence de la personne et/ou de son avocat à l'audience;

- la communication des pièces du greffe aux personnes présentes;

- le lieu où la séance se déroule: tribunal, hôpital ou visioconférence. Deux problèmes surgissent immédiatement: la résonance de la démarche judiciaire et de son décorum face à des personnes fragilisées, la difficulté pour les juges et les avocats d'exercer leurs missions dans un contexte inhabituel qui requiert certainement une formation spécifique à venir. Ils sont cependant compensés par le fait indiscutable que la nouvelle loi respecte mieux les droits des personnes et leur fragilité si elle se déroule selon un code usuel des «bonnes pratiques». Et d'ailleurs, au moment où nous parlons, contre toute attente au moment de sa promulgation, l'application de la loi, hormis la surcharge de travail qu'elle a occasionnée (tout le monde est d'accord sur ce point!), n'a pas entraîné de graves désordres ni créé d'incidents gravement dommageables pour les patients.

En cas d'hospitalisation d'office, l'UNAFAM et beaucoup de soignants n'admettaient pas que le préfet ait toujours le dernier mot. Ce n'est plus le cas avec la nouvelle loi : les pouvoirs du préfet se sont trouvés dans certains cas réduits au bénéfice du JLD.

Les patients que l'on dit en état de «dangerosité potentielle présumée» font l'objet de trois dispositions spécifiques :

- les patients déclarés irresponsables pénalement à la suite d'un délit et admis en soins psychiatriques par décision du préfet;

- les patients hospitalisés en unité pour malades difficiles (UMD) par décision du préfet;

- les patients admis en soins psychiatriques et ayant depuis moins de dix ans soit bénéficié de l'irresponsabilité pénale soit passé au moins un an en UMD.

L'UNAFAM s'est opposée à la notion de «dangerosité présumée» en particulier aux seules fins d'une hospitalisation en UMD. Malheureusement, le législateur a maintenu le principe d'une procédure spécifique en cas de passage en UMD même s'il a concédé un «droit à l'oubli» après dix ans. Reste que ces UMD sont un point qu'il faudrait aborder avec beaucoup de circonspection tant elles relèvent de situations d'une extrême complexité.

La nouvelle loi oblige les établissements de santé à conclure des conventions avec les représentants de l'État, des collectivités locales et les ARS. Elle stipule que les ARS doivent veiller à la qualité et à la coordination des actions de soutien et d'accompagnement des familles et des aidants de personnes malades menées par les établissements de santé et par les associations comme l'UNAFAM, agrées pour représenter les usagers dans les instances hospitalières ou de santé publique. Ce dernier point est évidemment une revendication de longue date de l'UNAFAM.

Ainsi, l'UNAFAM a tenté lors du projet menant à cette loi de 2011 de défendre ses revendications et de limiter des dispositions excessives. Elle considère que de nombreux points de la loi ont amélioré la situation des personnes malades et de leur entourage. Elle espère voir se généraliser des bonnes pratiques et manifeste son souci de voir disparaître toutes formes de maltraitance dans les applications de cette loi, en particulier à l'occasion des audiences (problèmes du lieu et du secret médical). 


\section{L'UNAFAM et la loi du 27 septembre 2013 modifiant la loi du 5 juillet 2011}

À la suite de la remise en cause par le Conseil constitutionnel de certains points de la loi no $2011-803$ du 5 juillet 2011 sur les soins sans consentement en psychiatrie, avec obligation d'apporter des corrections (ou de voir ces points annulés) avant le $1^{\text {er }}$ octobre $2013^{4}$, les parlementaires ont d'abord entrepris un travail d'analyse', puis des auditions des principales parties prenantes à l'Assemblée nationale en juillet et au Sénat en septembre. L’UNAFAM a été associée à ces différentes étapes. Il en est résulté un projet de loi en juillet, puis deux passages devant l'Assemblée nationale et le Sénat et un texte définitif de la loi du 27 septembre $2013^{6}$.

Comme le montraient les différentes étapes, les modifications apportées vont dans le sens de certaines des améliorations souhaitées par l'UNAFAM et ses partenaires (Fédération nationale des associations d'usagers en psychiatrie et Conférence des présidents de commissions médicales d'établissements psychiatrique) sans qu'ils n'aient eu totalement satisfaction. Il faut noter que les modifications interviennent en deux temps. Certaines sont applicables immédiatement. D'autres n'entreront en vigueur qu'au $1^{\text {er }}$ septembre 2014.

Dès maintenant, on relève la suppression du statut juridique spécial des UMD: les conditions de sortie de soins sans consentement pour les patients ayant été en UMD étaient le point fort contesté par le Conseil constitutionnel. La loi fait totalement disparaître cette disposition particulière. Par ailleurs, l'UMD est considérée comme une unité de soins intensifs (comme il en existe en chirurgie) a expliqué le rapporteur. Aussi il a été retenu de ne plus donner de statut légal aux UMD.

Le régime spécial de levée de soins ne s'applique plus qu'aux patients ayant été déclarés irresponsables pénaux, ayant encouru pour les faits commis plus de cinq ou dix ans de réclusion, selon qu'il s'agit d'atteinte aux personnes ou aux biens. Par contre, l'UNAFAM regrette qu'il n'ait rien été fait pour réduire les risques d'arbitraire d'envoi en UMD sur la simple décision d'un seul médecin. Ce point avait été aussi relevé par le Conseil constitutionnel.

Est également prévue une autorisation de sorties de courtes durées non accompagnées de quarante-huit heures pour les personnes en régime d'hospitalisation sans consentement: c'est le rétablissement des permissions d'essai complétant le régime actuel des permissions accompagnées de douze heures. Elles se font sur demande au directeur par un psychiatre de l'établissement. Il n'est pas établi pour ces sorties de programme de soins. Si les soins résultent de la demande d'un tiers, le directeur d'établissement informe le tiers préalablement à la sortie. Ce doit être la première fois qu'on pense au tiers que sont souvent les familles! Si la personne est sous un régime de soins à la demande du représentant de l'État, celui-ci doit être prévenu au plus tard quarante-huit heures avant la date prévue mais doit répondre avant midi la veille du jour de la sortie. Auparavant il pouvait refuser au dernier moment. D'où des situations difficiles à gérer alors que tout était prêt pour la sortie.

Les députés, sénateurs et parlementaires européens sont habilités à visiter les établissements accueillant des personnes en régimes de soins sans consentement. Cela complète la liste déjà existante des personnes habilitées à faire ces visites dont les membres des commissions départementales des soins psychiatriques.

Une réflexion sera engagée pour examiner la dématérialisation du registre tenu par chaque établissement concernant les personnes sous régime de soins sans consentement.

Enfin, le certificat médical dit «de huitaine» est supprimé.

$\mathrm{Au} 1^{\text {er }}$ septembre 2014, les audiences se tiendront dans une salle spécialement aménagée dans l'enceinte de l'établissement de santé ou, éventuellement, d'un établissement voisin du ressort du même tribunal de grande instance. Les audiences d'appel seront maintenues à la cour d'appel. L'utilisation de la visioconférence sera supprimée. L'UNAFAM demandait son maintien en cas de circonstances exceptionnelles (congères de neige interdisant les déplacements des magistrats, par exemple). Mais la nouvelle loi dit que des circonstances exceptionnelles pourront être admises pour des saisines tardives. On peut en conclure que des saisines faites dans les temps, dont l'audience serait contrariée pour des raisons exceptionnelles, seront aussi admises sans entraîner automatiquement la levée de la mesure d'hospitalisation.

Le délai de saisine du juge sera raccourci : le juge devra être saisi dans les huit jours pour une audience dans les douze jours (au lieu de quinze actuellement). Sur ce point, l'UNAFAM demandait que le tiers à l'origine des soins à la demande d'un tiers soit systématiquement invité à être entendu par le juge. Mais comme aucune instruction ne peut être donnée à un magistrat, la formulation fut que «lors de sa convocation à l'audience, le tiers fasse l'objet d'une attention particulière afin de ne pas l'exposer à l'éventuelle vindicte de la personne». Cette demande n'a pas été retenue.

Un délai imposé de quatre jours sera imposé entre la transmission du dossier au juge et l'audience. Ceci permettra-t-il aux juges de mieux penser à la convocation du tiers? La question reste ouverte aujourd'hui...

4. CC, déc. $\mathrm{n}^{\circ}$ 2012-235 QPC du 20 avril 2012, Association Cercle de réflexion et de proposition d'actions sur la psychiatrie.

5. D. Robiliard, Rapport fait au nom de la commission des affaires sociales sur la proposition de loi relative aux soins sans consentement en psychiatrie, $\mathrm{n}^{\circ}$ 1284, enregistré à la présidence de l'Assemblée nationale le 17 juillet 2013.

6. Loi n 2013-869 du 27 septembre 2013 modifiant certaines dispositions issues de la loi no $2011-803$ du 5 juillet 2011 relative aux droits et à la protection des personnes faisant l'objet de soins psychiatriques et aux modalités de leur prise en charge, JORF, 29 septembre 2013 , p. 16230. 
Il y aura obligation de l'assistance ou de la représentation par un avocat. Dans la quasi-totalité des cas, ce seront des avocats commis d'office ou des personnes relevant de l'aide juridictionnelle. Le paiement par dossier étant peu élevé, pour le fonctionnement de ces dispositions il parait nécessaire à l'UNAFAM que les frais de déplacement de ceux-ci soient pris en compte.

Enfin, lorsque l'hospitalisation devra être poursuivie, il ne faudra plus un avis conjoint de deux psychiatres de l'établissement mais l'avis motivé d'un seul psychiatre.

En guise de conclusion - provisoire -, il est incontestable qu'il y a eu une prise en compte des demandes de l'UNAFAM, d'autant plus qu'elles étaient collégiales. Par contre, trois points restent encore à éclaircir :

- réduire l'arbitraire de l'entrée en UMD;

- obtenir à l'audience une prise en compte systématique du point de vue de la famille quand elle le souhaite et sous la forme la plus opportune;
- réformer en psychiatrie la notion de personne de confiance (citée comme accompagnant dans les permissions accompagnées de douze heures) qui peut ouvrir la porte à bien des abus sur des personnes dont la volonté est gravement affectée. Cette question est aussi portée collégialement avec la Fédération nationale des associations d'usagers en psychiatrie et la Conférence des présidents de commissions médicales d'établissements psychiatriques.

Ces différentes questions ont été soulevées lors des auditions parlementaires. Il nous a été répondu que la mission du député Robiliard continuait (son rapport n'était qu'un rapport d'étape), en vue d'un volet psychiatrie dans la future loi de santé. La loi du 27 septembre 2013 avait pour but premier de réagir aux remarques du Conseil constitutionnel dans le délai imparti tout en en profitant pour apporter déjà un certain nombre d'améliorations. Mais le travail n'est pas fini, et l'UNAFAM reste bien sûr vigilante quant à l'application de cette loi et à ses évolutions futures. 